\title{
Influência do nível hidrológico sobre a dieta de Leporinus reinhardtii (Characiformes, Anostomidae) em um reservatório do semiárido brasileiro
}

\author{
Tatiane N. Medeiros ${ }^{1,2}$, Aline A. F. Rocha ${ }^{1,2}$, Natália C. L. Santos ${ }^{3} \&$ William Severi $^{1}$
}

\author{
1. Laboratório de Ictiologia, Departamento de Pesca e Aquicultura, Universidade Federal Rural de Pernambuco, Rua Dom Manoel de Medeiros, Dois Irmãos, 52171-900 Recife, PE, Brasil. \\ (tnmedeiros@hotmail.com; wseveri@depaq.ufrpe.br) \\ 2. Programa de Pós-graduação em Recursos Pesqueiros e Aquicultura, Departamento de Pesca e Aquicultura, Universidade Federal Rural de Pernambuco, Rua Dom Manoel de Medeiros, \\ Dois Irmãos, 52171-900, Recife, PE, Brasil. (alinerochabio@hotmail.com) \\ 3. Programa de Pós-graduação em Ecologia de Ambientes Aquáticos Continentais, Universidade Estadual de Maringá, Av. Colombo, 87020-900 Maringá, PR, Brasil. (nathy lacerda@gmail.com)
}

\begin{abstract}
Influence of the hydrological level on the diet of Leporinus reinhardtii (Characiformes, Anostomidae) in a semi-arid Brazilian reservoir. The Neotropical region presents a high number of freshwater fish species, whose capability to explore different kinds of food is particularly evident in reservoirs, and reflects a considerable feeding plasticity. Characiformes is the most diversified fish order in the region, and Anostomidae one of its most speciose families, evidencing Leporinus and its 80 valid species. Although its species are mainly considered omnivorous, some present herbivorous diet, and the food plasticity of the genus is a common feature in environments subject to a seasonal variation of water level. In the present work, the diet composition of Leporinus reinhardtii Lütken, 1875 in the Sobradinho reservoir and the influence of its seasonal water level variation is analyzed along a three-year period (2006-2009). Among the 876 individuals analyzed, 302 were collected in the dry phase, and 574 in the flooding phase. The diet was composed of 47 items, grouped into nine categories: Mollusks, Microcrustaceans, Insects, Insect (parts), Chironomidae, Plants, Seeds, Sediment and Others. Chironomidae represented a mean of $60 \%$ of total weight of all food categories, followed by Seeds with $25 \%$ and the remaining categories with less than $10 \%$. In the flooding phase, Seeds prevailed in the diet in the lentic and transition stretches, Chironomidae was most represented in the lotic one, and the remaining categories represented less than $20 \%$ in all stretches at this phase. In the dry phase, the prevailing items differed, with a higher dominance of Insetcs in the lentic stretch, mainly Coleoptera, and Plants in the lotic one, with greater participation of aquatic macrophytes, filamentous algae, Chlorophyceae and the diatom Oscillatoria. In the transition stretch, Microcrustaceans dominated in the diet, differing from the other stretches. The diet of $L$. reinhardtii was characterized by low values of frequence of occurrence for all food categories (mostly inferior to 50\%), and high relative abundance ( $>80 \%$ ) of all categories. Niche breadth was restricted in the different stretches in both periods, somewhat higher during flooding, due to a greater item diversity, particularly in the lotic stretch. Leporinus reinhardtii presents an omnivorous habit with specialized behaviour in the Sobradinho reservoir, whose prevailing food categories in the diet depend on the reservoir level, as well as the environmental characteristics of each stretch, thus reflecting a narrow trophic niche breadth for the species.
\end{abstract}

KEYWORDS. São Francisco River, omnivory, piau.

RESUMO. A Região Neotropical possui um elevado número de espécies de peixes de água doce, cujas adaptações para explorar diferentes tipos de alimento é particularmente evidente em reservatórios, refletindo numa considerável plasticidade alimentar. Characiformes é a mais diversificada ordem de peixes da região, tendo Anostomidae como uma das famílias mais especiosas, com destaque para Leporinus, com cerca de 80 espécies válidas. Embora suas espécies sejam consideradas primordialmente onívoras, algumas apresentam dieta herbívora, e a plasticidade alimentar do gênero é uma característica comum em ambientes sujeitos a variação sazonal de nível da água. No presente trabalho, são analisadas a composição da dieta de Leporinus reinhardtii Lütken, 1875 no reservatório de Sobradinho e a influência da variação sazonal de seu nível sobre a mesma ao longo de três ciclos anuais (2006-2009). Dentre os 876 indivíduos analisados, 302 foram coletados no período de seca e 574 no de cheia. A dieta foi composta por 47 itens, agrupados em nove categorias: Moluscos, Microcrustáceos, Insetos, Insetos (partes), Chironomidae, Vegetais, Sementes, Sedimento e Outros. Chironomidae representou em média $60 \%$ do peso total das categorias alimentares, seguido de Sementes com $25 \%$ e as demais categorias com menos de $10 \%$. No período de cheia, nos trechos lêntico e de transição, predominaram Sementes na dieta, tendo Chironomidae sido mais representativa no trecho lótico, e as demais categorias não ultrapassando $20 \%$ de participação nos três trechos neste período. No período de seca, houve diferença nos itens predominantes, com predominância de Insetos no trecho lêntico, principalmente Coleoptera, e de Vegetais no lótico, com maior participação de macrófitas aquáticas, algas filamentosas, clorofíceas e da diatomácea Oscillatoria. Já no trecho de transição, Microcrustáceos dominaram na dieta, diferindo dos demais trechos. A dieta de L. reinhardtii se caracterizou por baixos valores de frequência de ocorrência de todas as categorias alimentares (predominantemente inferiores a 50\%) e elevada abundância relativa $(>80 \%)$ de diferentes categorias. A amplitude de nicho foi restrita nos diferentes trechos nos dois períodos, sendo ligeiramente mais elevada na cheia, devido à maior diversidade de itens, em particular no trecho lêntico. Leporinus reinhardtii possui hábito onívoro com comportamento especialista no reservatório de Sobradinho, cujas categorias predominantes da dieta dependem do regime hidrológico do reservatório e das características ambientais de cada trecho, refletindo numa amplitude restrita de nicho trófico para a espécie.

PALAVRAS-CHAVE. Rio São Francisco, onivoria, piau.

A Região Neotropical é conhecida pelo elevado número de espécies de peixes de água doce, que se reflete nas adaptações desta ictiofauna para explorar diferentes tipos de alimento (AgostinHo et al., 2007). Tais adaptações são evidentes em reservatórios, ambientes sujeitos às alterações decorrentes da modificação do meio natural original de rio para lago, nos quais as espécies dotadas de maior plasticidade alimentar são melhor sucedidas na colonização e na exploração dos recursos alimentares disponíveis (HAHN \& FUGI, 2007).

Characiformes é a mais diversificada ordem de peixes da Região Neotropical, com mais de 1700 espécies distribuídas em aproximadamente 270 gêneros e 18 famílias (Nelson, 2006). Entre elas, destacam-se aquelas pertencentes a Anostomidae, amplamente distribuídas nas porções cis-andina e trans-andina dessa região, contando 
com cerca de 140 espécies, incluídas em 14 gêneros (Garavello \& Britski, 2003; Sidlauskas \& VARI, 2008). Leporinus é o gênero mais especioso da família, com cerca de 80 espécies válidas (BRITSKi et al., 2012).

Embora espécies de Leporinus sejam primordialmente consideradas como onívoras (GERKING, 1994; Durães et al., 2001; Albrecht \& PellegriniCARAmaschi, 2003; Balassa et al., 2004), uma dieta herbívora também tem sido reportada para diversas espécies (Goulding, 1980; FERREIRA, 1984; BENNEMANn et al., 2000).

Um caráter predominantemente herbívoro é reflexo da história de vida de cada espécie, além de suas características morfofisiológicas, estreitamente associadas a adaptações evolutivas. Entretanto, uma elevada flexibilidade alimentar parece ser característica das espécies de Leporinus, notadamente em ambientes alterados, como reservatórios (AgostinHo et al., 2007), em particular naqueles de acumulação, sujeitos a ampla variação de nível da água ao longo do ano, em decorrência do pulso de inundação.

Ambientes sujeitos a pulsos de inundação são alagados periodicamente e enriquecidos com nutrientes que elevam a produção primária do ecossistema, consequentemente, afetando sua estrutura trófica. Alterações físico-químicas durante os pulsos afetam a biota aquática, influindo na qualidade e disponibilidade de recursos alimentares e provocando mudanças morfológicas, fisiológicas, anatômicas e comportamentais associadas à dieta de peixes e outros organismos (JUNK et al., 1989).

A bacia do rio São Francisco é uma das bacias brasileiras com o maior número de reservatórios (7), dentre os quais se destaca o reservatório de Sobradinho, sujeito a uma ampla variação sazonal de seu nível hidrológico, por funcionar como regulador do abastecimento de água da cascata de reservatórios de seu trecho submédio. A bacia abriga seis espécies de Leporinus (BucKuP et al., 2007), sendo que o conhecimento sobre a dieta destas é limitado a Leporinus reinhardtii Lütken, 1875 (Pompeu \& GodinHo, 2003; Alvim \& Peret, 2004) e Leporinus piau Fowler, 1941(Gomes \& Verani, 2003), em estudos realizados no reservatório de Três Marias (alto São Francisco) e em lagoas marginais do trecho médio da bacia. A despeito da abundância de $L$. reinhardtii na comunidade de peixes do reservatório de Sobradinho, onde a mesma tem papel relevante na pesca comercial, inexistem dados sobre a ecologia trófica da espécie e/ou sobre sua participação na partilha de recursos dentre outros anostomídeos, que subsidiem ações de manejo e ordenamento pesqueiro.

Neste contexto, o presente trabalho pretende (a) caracterizar a composição da dieta da espécie em diferentes porções do reservatório de Sobradinho e (b) avaliar a influência da variação de nível do reservatório sobre a mesma ao longo de três ciclos anuais.

\section{MATERIAL E MÉTODOS}

Área de Estudo. O reservatório de Sobradinho foi formado pelo represamento do rio São Francisco, a montante da cidade de Sobradinho, estado da Bahia

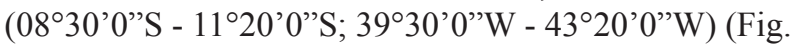
1). Construído em 1979 com a finalidade de regularizar a vazão do rio São Francisco, Sobradinho é o terceiro maior reservatório hidrelétrico do Brasil em volume armazenado, com $34 \times 10^{9} \mathrm{~m}^{3}$, e aproximadamente 420.000 ha de área, a qual pode ser reduzida em até $70 \%$ no período de seca. Possui uma extensão longitudinal de aproximadamente $360 \mathrm{~km}$, tendo como limite de inundação a cidade de Xique-Xique (BA), e sua bacia de captação tem o rio São Francisco como principal contribuinte, aliado a diversos rios intermitentes, com vazão sazonal apenas no período chuvoso, cujos vales inundados formaram as reentrâncias de ambas as margens (RocHA et al., 2011).

O reservatório possui três trechos (lótico, de transição e lêntico, sensu THORNTon, 1990), com características distintas de velocidade da água, declividade das margens e área alagada em função da variação sazonal de nível da água, conforme os limites constantes de Pinto et al. (2011) (Fig. 1).

Os períodos de seca (meses de amostragem de novembro, janeiro e setembro) e cheia (março, maio e julho) foram caracterizados em função do nível do reservatório, expresso em cota (metros acima do nível do mar), resultante da variação da vazão afluente e da operação da usina hidrelétrica durante o período de estudo (PinTo et al., 2011).

Procedimento em campo. As coletas foram realizadas bimestralmente em pontos localizados em cada um dos três trechos do reservatório, entre setembro de 2006 e outubro de 2009 . Foram utilizadas redes de espera com aberturas de malha variadas $(12,15,20,25,30$ e 35 $\mathrm{mm}$ entre nós adjacentes), lançadas sempre ao anoitecer e retiradas na manhã seguinte, com exposição aproximada de 12 horas. Após a retirada das redes, os exemplares foram devidamente identificados (BRITSKI et al., 1984), pesados (peso total em gramas) e medidos (comprimento padrão em mm), sacrificados através de choque térmico com gelo, acondicionados em caixas térmicas e transportados para o laboratório.

Procedimento em laboratório. Após a biometria, os indivíduos foram eviscerados para a retirada dos estômagos, os quais foram pesados $(0,001 \mathrm{~g})$, fixados em formol $4 \% \mathrm{e}$ acondicionados em frascos de polietileno, para posterior análise dos itens alimentares.

O conteúdo estomacal foi analisado e identificado sob estereomicroscópio, com o maior detalhamento taxonômico possível dos itens alimentares utilizando bibliografia especializada (BRITSKi et al., 1984; MerRitT \& Cummins, 1996; Sмith, 2001; Simone, 2006). Exemplarestestemunho foram depositados na Coleção de Peixes do Laboratório de Ictiologia do Departamento de Pesca e Aquicultura da UFRPE, em Recife (PE).

Tratamento dos dados. Foi utilizado o método de frequência de ocorrência dos itens alimentares (Fi) (ZAVALACAMin, 1996) e o método gravimétrico (Pi) (Hyslop, 1980). A dieta foi avaliada por trecho (lótico, transição e 


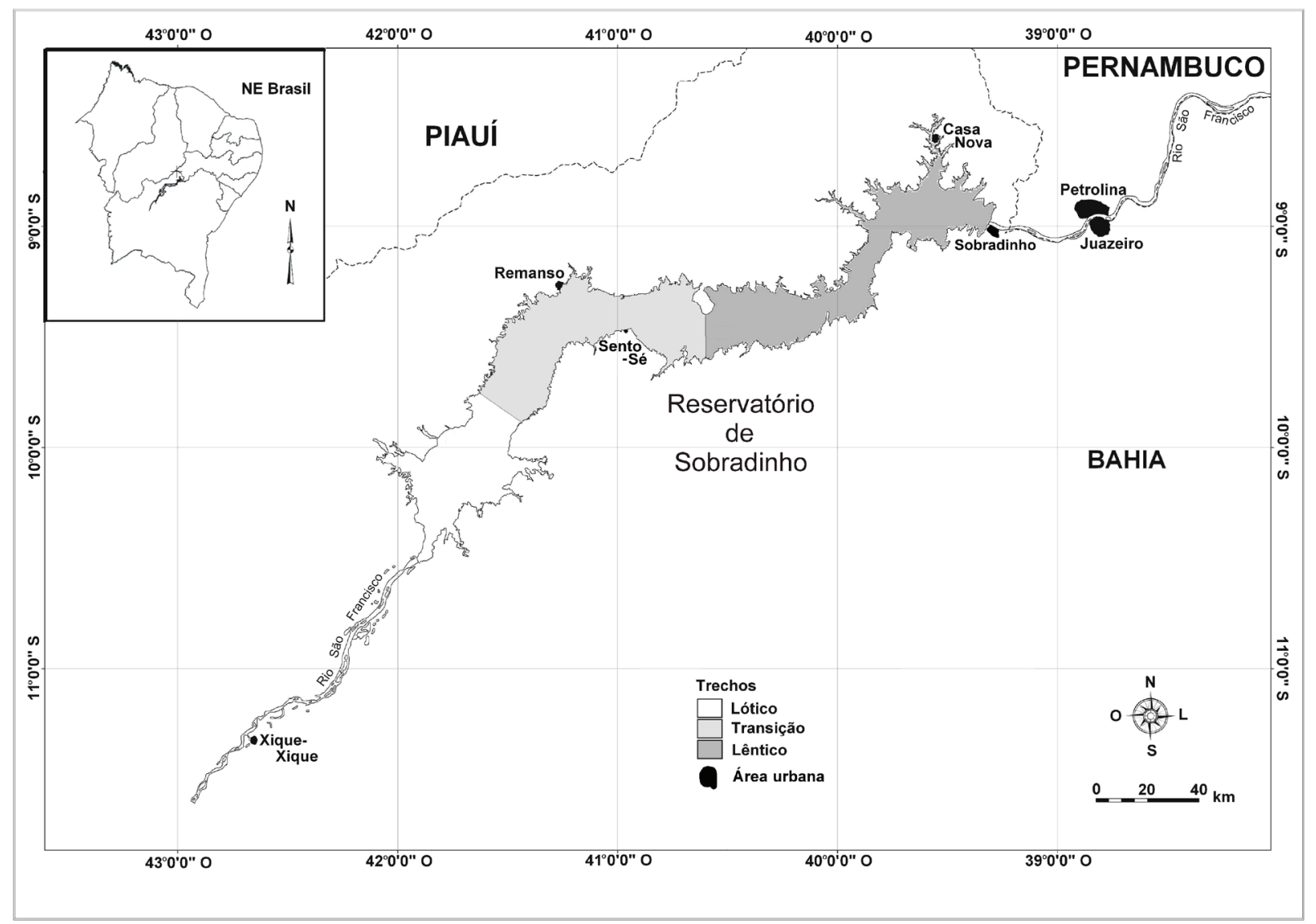

Fig. 1. Mapa do reservatório de Sobradinho, com indicação de seus diferentes trechos e localização na região Nordeste. Modificado de PINTo et al. (2011).

lêntico) e nível da água do reservatório (períodos de seca e cheia). A fim de visualizar as variações na composição da dieta entre os trechos amostrados foi realizado um escalonamento multidimensional não-métrico (nMDS) baseado na análise de cluster utilizando UPGMA, construído através da matriz de similaridade de BrayCurtis com o programa Primer 6.0.

A análise de similaridade (ANOSIM) foi usada para testar a hipótese nula (H0), a qual indica que não existe diferença significativa $(p>0,05)$ na composição da dieta entre os grupos formados na nMDS. O valor do R-estatístico varia de -1 a 1 , e quanto mais próximos a zero forem os valores, aceita-se HO (Clarke \& Gorley, 2006). Esta análise foi conduzida através do programa Primer 6.0.

Com a finalidade de avaliar o caráter generalista ou especialista da espécie, foi utilizada a representação gráfica de Costello (1990), modificada por AMUNDSEn et al. (1996), que considera a contribuição fenotípica entre (BPC) e dentre (WPC) os indivíduos, em relação à importância das presas em cada trecho, de acordo com o nível da água do reservatório.

A amplitude de nicho trófico (amplitude da dieta) foi estimada através do índice padronizado de Levins (KREBS, 1999), com o objetivo de demonstrar o seu grau de especialização. Este varia de 0, quando uma espécie consumiu somente um tipo de presa, a 1 , quando esta consumiu vários tipos de presas de forma similar. Para uniformização da medida do nicho trófico foi aplicada a fórmula de HuRLBERT (1978).

\section{RESULTADOS}

Foram analisados 876 indivíduos de Leporinus reinhardtii, 302 correspondentes ao período de seca e 574 ao de cheia. Um total de 54 itens foram registrados na dieta (Tab. I), agrupados em nove categorias: Moluscos, Microcrustáceos, Insetos, Insetos (partes), Chironomidae, Vegetais, Sementes, Sedimento e Outros.

Chironomidae representou em média $60 \%$ do peso total das categorias alimentares encontradas nos estômagos analisados, seguida de Sementes com 25\% (Fig. 2), tendo as demais categorias importância secundária na dieta, com participação inferior a $10 \%$.

Para o período de cheia, nos trechos lêntico e de transição, houve predominância de Sementes, enquanto Chironomidae foi a categoria mais representativa no trecho lótico, e as demais não ultrapassaram $20 \%$ nos três trechos neste período (Fig. 3). No período de seca, predominaram itens diferentes daqueles registrados no período de cheia, com predominância de Insetos no trecho lêntico, principalmente da ordem Coleoptera, e de Vegetais no trecho lótico, com maior participação de macrófitas aquáticas, algas filamentosas, clorofíceas e da diatomácea Oscillatoria. No trecho de transição, os Microcrustáceos 
Tab. I. Itens alimentares registrados para Leporinus reinhardtii Lütken, 1875 no período de seca e cheia em todo o período estudado no reservatório de Sobradinho, Brasil.

\begin{tabular}{|c|c|c|c|c|c|c|c|c|c|c|c|c|}
\hline \multirow{3}{*}{ ITENS ALIMENTARES } & \multicolumn{4}{|l|}{ Lótico } & \multicolumn{4}{|l|}{ Transição } & \multicolumn{4}{|l|}{ Lêntico } \\
\hline & seca & & cheia & & seca & & cheia & & seca & & cheia & \\
\hline & FO (\%) & $\mathrm{V}(\%)$ & FO (\%) & $\mathrm{V}(\%)$ & FO (\%) & V $(\%)$ & FO $(\%)$ & $\mathrm{V}(\%)$ & FO $(\%)$ & V $(\%)$ & FO $(\%)$ & $\mathrm{V}(\%)$ \\
\hline Nematoda & & & 1,052 & 0,003 & & & 2,298 & 0,013 & & & 2,222 & $<0,001$ \\
\hline Annelida & & & & & & & 1,149 & $<0,001$ & & & & \\
\hline Oligochaeta & & & & & & & 2,298 & 0,317 & & & & \\
\hline Moluscos & 1,408 & $<0,001$ & 1,052 & $<0,001$ & & & 1,149 & 0,003 & & & & \\
\hline Moluscos (partes) & 1,408 & $<0,001$ & 4,21 & $<0,001$ & & & & & & & & \\
\hline Bivalvia & & & 20,526 & 7,728 & & & 8,045 & 0,435 & & & 4,444 & $<0,001$ \\
\hline Bivalvia (partes) & 1,408 & $<0,001$ & 17,894 & 1,991 & & & 13,793 & $<0,001$ & & & & \\
\hline Corbicula fluminea & & & & & & & 6,896 & $<0,001$ & & & & \\
\hline Gastropoda & 1,408 & $<0,001$ & 3,684 & 0,007 & 2,857 & 0,228 & 5,747 & 0,02 & 1,886 & $<0,001$ & 6,666 & 3,818 \\
\hline Gastropoda (partes) & & & 2,105 & $<0,001$ & & & 4,597 & 0,013 & & & 4,444 & $<0,001$ \\
\hline Conchostraca & & & 4,21 & 0,005 & & & 17,241 & 10,95 & & & 15,555 & 0,415 \\
\hline Cladocera & 2,816 & $<0,001$ & 0,526 & $<0,001$ & 8,571 & 83,257 & 2,298 & $<0,001$ & & & & \\
\hline Ostracoda & 2,816 & $<0,001$ & 2,631 & 0,002 & 2,857 & $<0,001$ & 1,149 & 0,027 & & & 4,444 & 0,047 \\
\hline Insetos & & & 0,526 & 0,015 & & & 4,597 & 1,45 & 1,886 & $<0,001$ & & \\
\hline Insetos (partes) & 1,408 & 0,027 & 1,052 & 0,373 & & & 5,747 & 2,39 & & & 13,333 & 15,621 \\
\hline Estojo larval & & & 3,684 & 0,007 & & & 3,448 & 0,027 & & & 6,666 & 0,016 \\
\hline Pupa & & & 0,526 & 0,27 & & & 1,149 & 0,082 & 1,886 & 100 & 6,666 & 10,108 \\
\hline Coleoptera & 1,408 & 1,657 & & & & & & & & & & \\
\hline Coleoptera (larva) & & & 0,526 & 0,001 & 2,857 & $<0,001$ & & & & & & \\
\hline Coleoptera (larva) - Hydrophilidae & & & 1,052 & 0,003 & 2,857 & 0,457 & & & & & 4,444 & 0,084 \\
\hline Coleoptera (partes) & 1,408 & $<0,001$ & & & & & 1,149 & 0,193 & & & & \\
\hline Díptera (partes) & & & & & & & & & & & 2,222 & 0,011 \\
\hline Chironomidae (larva) & 4,225 & $<0,001$ & 38,947 & 83,674 & 11,428 & 8,234 & 9,195 & 3,506 & & & 17,777 & 2,409 \\
\hline Chironomidae (partes) & 1,408 & $<0,001$ & 2,105 & 0,008 & & & 2,298 & 0,01 & & & 11,111 & 2,65 \\
\hline Ablabesmyia & & & & & & & 1,149 & 0,003 & & & 6,666 & 0,016 \\
\hline Chironomus & & & & & & & & & & & 2,222 & 0,134 \\
\hline Coebtanypes & & & & & & & & & & & 2,222 & 0,005 \\
\hline Dicrotendipes & & & 0,526 & 0,003 & & & 6,896 & 0,072 & & & 13,333 & 1,465 \\
\hline Goeldichironomus & & & 0,526 & 0,005 & & & & & & & 2,222 & 0,297 \\
\hline Polypedilum & & & & & 2,857 & 0,457 & 1,149 & $<0,001$ & & & & \\
\hline Tanytarsus & & & & & & & 1,149 & $<0,001$ & & & & \\
\hline Ephemeroptera & & & 1,578 & 0,003 & & & 4,597 & 0,02 & & & 2,222 & 0,005 \\
\hline Polymirtacyidae & & & & & 2,857 & & & & & & & \\
\hline Ephemeroptera (larva) & & & & & & & & & & & 2,222 & 0,112 \\
\hline Ephemeroptera (partes) & & & 1,052 & 0,327 & 2,857 & $<0,001$ & 1,149 & $<0,001$ & & & & \\
\hline Hemiptera (partes) & & & & & & & 1,149 & $<0,001$ & & & & \\
\hline Odonata & 1,4081 & $<0,001$ & & & & & 0 & 0 & & & & \\
\hline Libellulidae & & & & & & & 0 & 0 & & & & \\
\hline Anysoptera (partes) & & & 0,526 & $<0,001$ & & & 0 & 0 & & & & \\
\hline Zygoptera & & & & & & & 1,149 & 0,006 & & & & \\
\hline Trichoptera & & & 2,631 & 0,017 & & & 0 & 0 & & & 4,444 & 0,084 \\
\hline Hidroptilidae & & & & & & & 0 & 0 & & & & \\
\hline Polycentropodidae & & & 0,526 & $<0,001$ & & & 0 & 0 & & & & \\
\hline Vegetais & 19,718 & 20,546 & 2,105 & $<0,001$ & & & 13,793 & 1,855 & & & 4,444 & $<0,001$ \\
\hline Macrófitas aquáticas & 7,042 & $<0,001$ & 1,052 & 0,378 & & & 1,149 & $<0,001$ & 7,547 & $<0,001$ & 2,222 & 9,794 \\
\hline Algas filamentosas & 5,633 & 0,027 & 3,157 & $<0,001$ & & & 0 & 0 & 1,886 & $<0,001$ & & \\
\hline Clorofícea & 1,408 & $<0,001$ & & & & & 0 & 0 & & & & \\
\hline Oscillatoria & 1,408 & 0,359 & & & & & 0 & 0 & & & & \\
\hline Sementes & 45,07 & 3,148 & 13,157 & 4,959 & 40 & 7,593 & 67,816 & 78,071 & 30,188 & $<0,001$ & 46,666 & 52,903 \\
\hline Sementes (partes) & 9,859 & $<0,001$ & 1,578 & $<0,001$ & 17,142 & $<0,001$ & 4,597 & $<0,001$ & & & 4,444 & $<0,001$ \\
\hline Matéria orgânica & 1,408 & $<0,001$ & 0,526 & $<0,001$ & & & & & & & & \\
\hline Sedimento & 7,042 & 0,11 & 0,526 & $<0,001$ & & & & & 3,773 & $<0,001$ & 2,222 & $<0,001$ \\
\hline Material digerido NI & 11,267 & 74,123 & 1,578 & 0,212 & & & 5,747 & 0,525 & & & 4,444 & $<0,001$ \\
\hline Estômagos c/ alimento & 71 & & 190 & & 35 & & 87 & & 53 & & 45 & \\
\hline
\end{tabular}

dominaram na dieta, diferindo dos demais trechos (Fig. 3). A análise de nMDS evidenciou diferença na composição da dieta da espécie entre os trechos apenas no período de seca (Fig. 4). Embora não tenha sido evidenciada diferença significativa de forma geral entre os períodos amostrados (ANOSIM, $\mathrm{p}>0,05$ ), foi detectada diferença significativa na dieta considerando os trechos amostrados, principalmente entre os lótico e de transição, bem como lótico e lêntico (ANOSIM, p <0,05) (Tab. II).

A dieta de $L$. reinhardtii se caracterizou por 


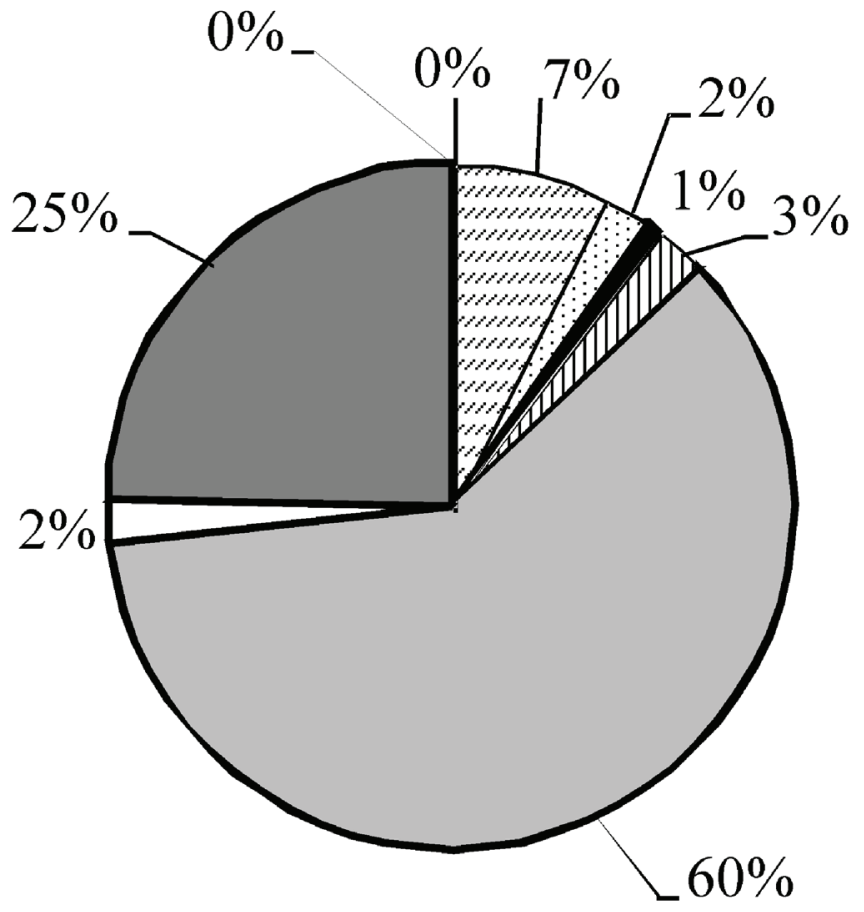

øOutros

Moluscos

๑Microcrustáceos

DInsetos

⿴囗十 Insetos (partes)

口Chironomidae

$\boldsymbol{\square}$ Vegeta is

口Sementes

BSedimento

Fig. 2. Participação relativa em peso das categorias alimentares na dieta de Leporinus reinhardtii Lütken, 1875 em todo o período de estudo no reservatório de Sobradinho.

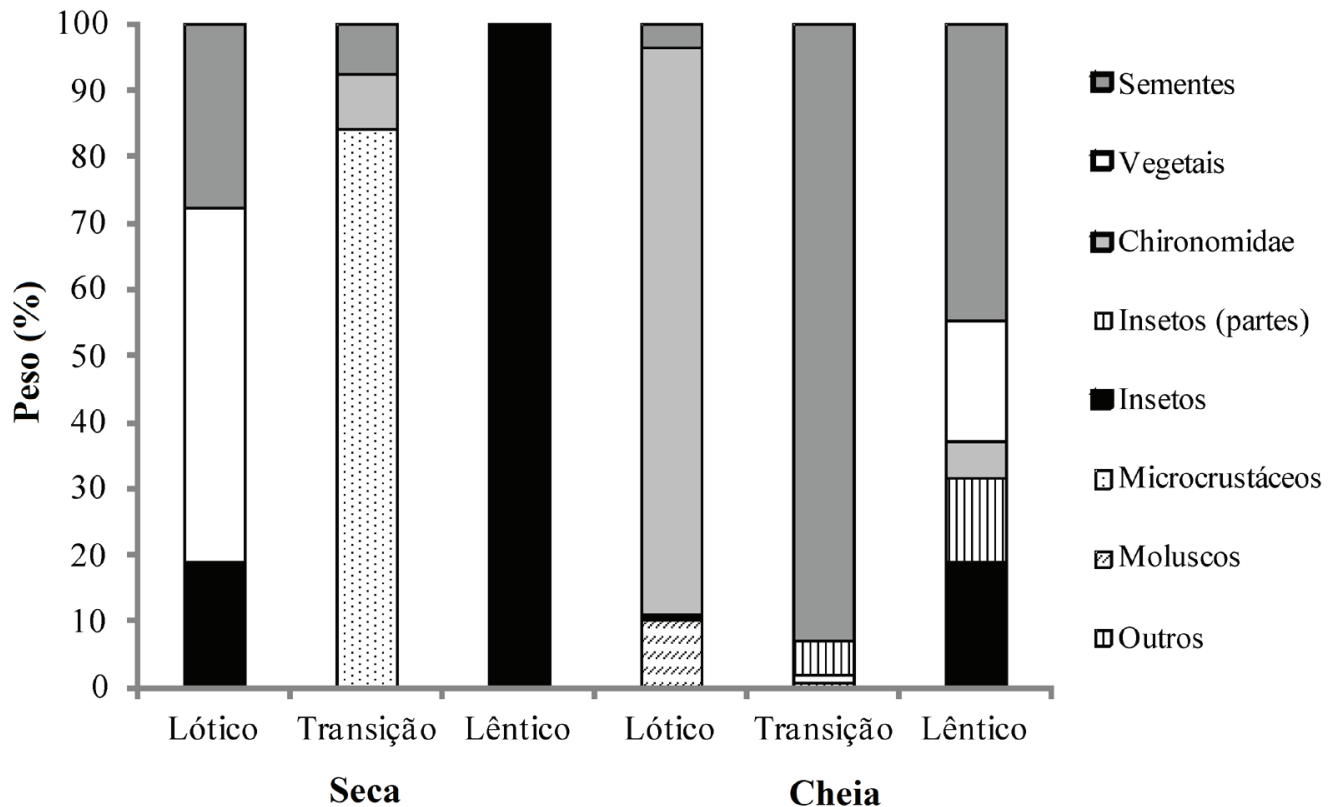

Fig. 3. Participação relativa em peso das categorias alimentares de Leporinus reinhardtii Lütken, 1875 nos três trechos amostrados no reservatório de Sobradinho, nos períodos de seca e cheia.

valores de frequência de ocorrência de todas as categorias alimentares em sua maioria inferiores a $50 \%$ e abundância relativa superior a $80 \%$ para diferentes categorias (Fig. 5). No período de seca, apenas Sementes apresentou FI $\geq 50 \%$, com abundância elevada de diferentes categorias entre os trechos, sendo Vegetais no lótico (>80\%), Microcrustáceos no de transição (>80\%) e Insetos no lêntico (100\%). No período de cheia, além de valores de $\mathrm{FI}<50 \%$, as categorias dominantes entre os trechos foram Chironomidae no lótico $(>80 \%)$ e Sementes no de transição $(\approx 80 \%)$ e lêntico $(\approx$ $50 \%)$.

A amplitude de nicho verificada foi restrita nos 


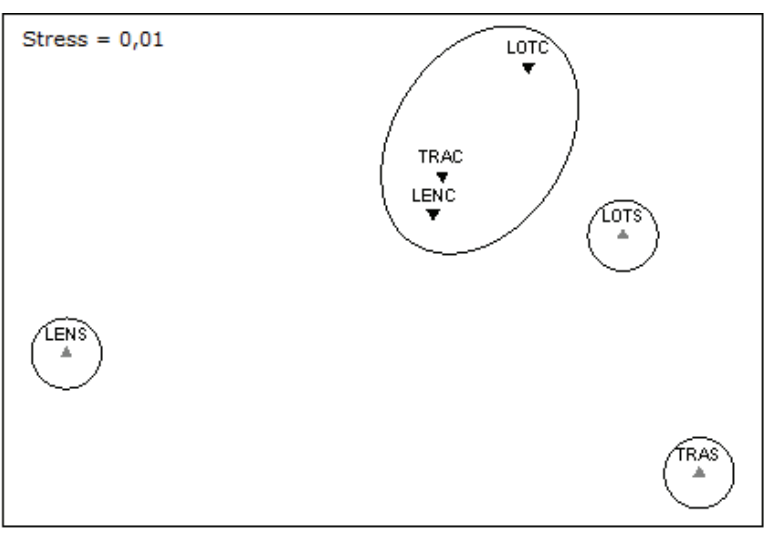

Fig. 4. Representação gráfica da análise de escalonamento multidimensional não-métrico (nMDS) da dieta de Leporinus reinhardtii Lütken, 1875 entre os períodos de coleta e trechos do reservatório de Sobradinho (BA, Brasil). Stress $=0,01$ (LENC, lêntico cheia; LENS, lêntico seca; LOTC, lótico cheia; LOTS, lótico seca; TRAC, transição cheia; TRAS, transição seca).

Tab. II. Análise de similaridade (ANOSIM) entre os trechos amostrados no reservatório de Sobradinho.

\begin{tabular}{lcc}
\hline \multirow{2}{*}{ Grupos } & \multicolumn{2}{c}{ Significância } \\
\cline { 2 - 3 } & R-estatístico & Nível \\
\hline Lótico, Transição & 0,133 & 0,001 \\
Lótico, Lêntico & 0,078 & 0,027 \\
\hline Transição, Lêntico & $-0,017$ & 0,712 \\
\hline
\end{tabular}

diferentes trechos nos dois períodos, sendo ligeiramente mais elevada na cheia, devido à maior diversidade de itens encontrada neste período, em particular no trecho lêntico (Fig. 6).

\section{DISCUSSÃO}

Em reservatórios, os recursos alimentares consumidos por peixes são geralmente originados do próprio ecossistema aquático, como invertebrados, incluindo insetos aquáticos e zooplâncton, além de detritos orgânicos e peixes (AGostinHo et al., 2007), o que determina o hábito onívoro de muitas espécies.

O caráter onívoro foi evidente na dieta de $L$. reinhardtii no reservatório de Sobradinho, o qual pode ser atribuído à oscilação periódica de nível do reservatório e à heterogeneidade de habitats explorados, que propiciam uma considerável disponibilidade de recursos no ambiente.

O hábito onívoro foi registrado para outras espécies de Leporinus. DuRÃes et al. (2001) analisaram a dieta de quatro espécies do gênero durante a fase de enchimento do reservatório Nova Ponte, no sudeste do Brasil, e concluíram que L. amblyrhynchus Garavello \& Britski, 1987 é uma espécie especialista, com alimentação constituída basicamente de insetos bentônicos, enquanto $L$. elongatus Valenciennes, 1849 e L. friderici (Bloch, 1794) foram caracterizadas como onívoras e L. octofasciatus Steindachner, 1917 como herbívoro. Albrecht \& Pellegrini-Caramaschi (2003) também registraram a onivoria de L. taeniofasciatus Britski, 1997 no alto rio
Tocantins. Na área de influência do reservatório do MansoMT, BALASSA et al. (2004) estudaram quatro espécies do gênero e observaram a onivoria em todas elas: Leporinus sp., L. elongatus, L. striatus Kner, 1858 e L. friderici. Por sua vez, Gomes \& Verani (2003) classificaram L. piau no reservatório de Três Marias como herbívoro, embora com tendência à onivoria.

Em regiões tropicais, sujeitas a amplas variações sazonais no nível d'água, a dieta de peixes é fortemente influenciada por mudanças no regime hidrológico, o que se refletiu na predominância de diferentes itens nos períodos de seca e cheia para o reservatório de Sobradinho.

No período seco, os insetos foram mais explorados no trecho lêntico, no qual as sementes também apresentaram participação expressiva na dieta. Tal fato pode estar relacionado à temperatura e transparência mais elevadas em trechos lênticos, que favorecem uma maior produtividade do ecossistema (Petry et al., 2011), com benefício para níveis tróficos mais elevados, como os insetos aquáticos. Áreas com maior produtividade apresentam refúgios que podem ser explorados por espécies distintas, capazes de utilizá-las para a manutenção de populações viáveis durante períodos com escassez de alimento (OliveIRA \& GoularT, 2000).

O reservatório de Sobradinho apresenta uma extensa região litorânea, principalmente nos trechos lótico e de transição, devido à menor profundidade do talvergue e ao menor declive das margens, com a presença de bancos de macrófitas que servem de refúgio e local de alimentação para a espécie em estudo. Servem, ainda, como substrato para muitos invertebrados (TAKEDA et al., 2003), como os microcrustáceos por exemplo, que dominaram na dieta no trecho de transição no período de seca. A elevada ocorrência de vegetais no trecho lótico neste mesmo período, pode estar associada à esta extensa região litorânea.

Em períodos de águas baixas, a disponibilidade de alimento torna-se mais restrita (GouldiNG, 1980), justificando a maior composição de vegetais na dieta da espécie em estudo, visto que a disponibilidade de insetos torna-se limitada. Além disso, a menor profundidade da água nesse período e maior penetração de luz estimulam a proliferação de macrófitas aquáticas.

No período de cheia, a notável presença de sementes foi evidente tanto para o trecho lêntico, como para o de transição. As inundações periódicas em sistemas neotropicais ocasionam o alagamento de extensas faixas de terra, expandindo sazonalmente o ambiente aquático, permitindo a incorporação de organismos alóctones, como insetos e material vegetal, considerados fontes alimentares importantes para os peixes (Lowe-McConnell, 1999). Entretanto, elevações drásticas e repentinas do volume da água associadas a uma maior correnteza, carreiam sedimento e organismos, tornando-os disponíveis para a alimentação de peixes (PETRY et al., 2011). Tal fato pode ser corroborado no trecho lótico de Sobradinho durante o período da cheia, pela maior participação de quironomídeos e moluscos na dieta de $L$. reinhardtii. 

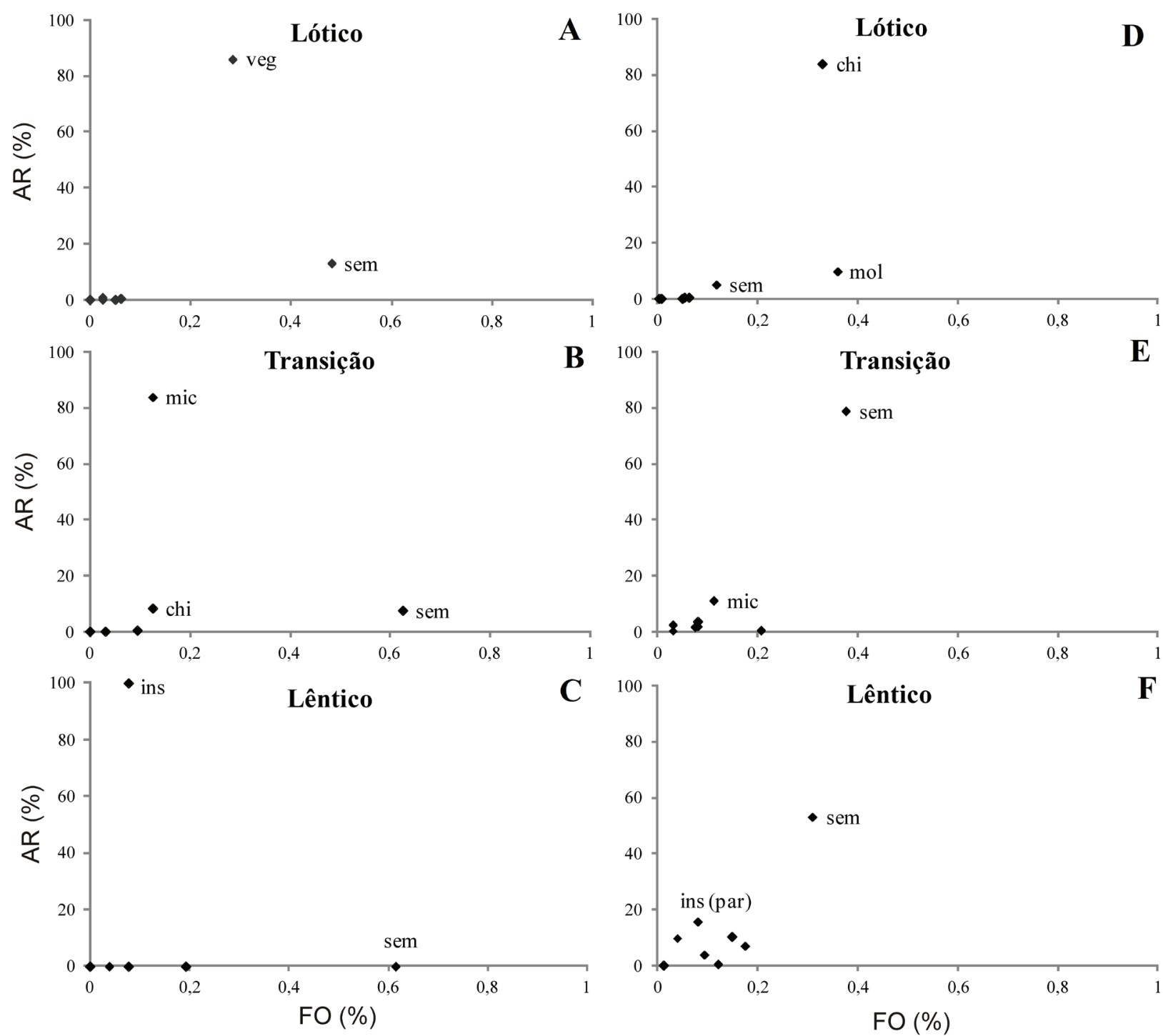

Fig. 5. Variação da frequência de ocorrência (FO) e da abundância relativa (AR) das categorias alimentares de Leporinus reinhardtii nos três trechos amostrados nos períodos de seca $(\mathrm{A}, \mathrm{B}, \mathrm{C})$ e cheia $(\mathrm{D}, \mathrm{E}, \mathrm{F})$ no reservatório de Sobradinho.

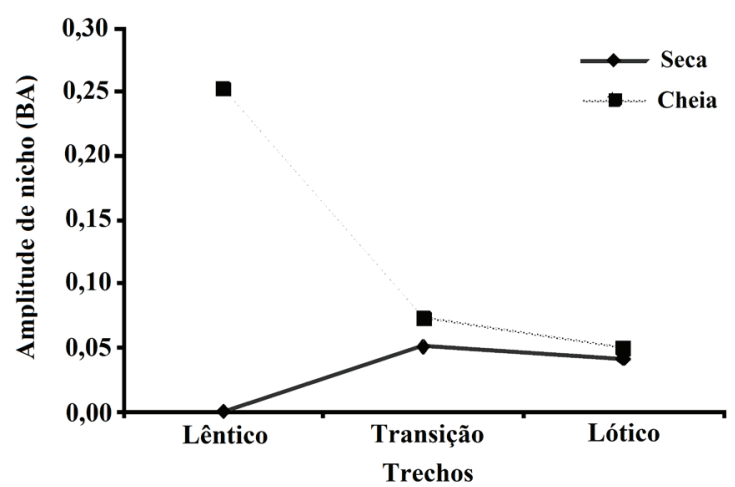

Fig. 6. Valores de amplitude de nicho alimentar de Leporinus reinhardtii nos três trechos amostrados no reservatório de Sobradinho, nos períodos de seca e cheia.

Em estudo sobre macroinvertebrados bentônicos realizado concomitamente com o presente trabalho (BORGES et al., 2010), foi registrada a predominância de insetos aquáticos na comunidade bentônica da zona litoral do reservatório de Sobradinho, tanto no período seco quanto no chuvoso. O aumento da participação de insetos aquáticos, principalmente larvas de Chironomidae, na dieta de $L$. reinhardtii no período de cheia, pode estar associado à elevação do nível da água, da inundação destas áreas e aumento da velocidade da correnteza, os quais podem ter contribuído para a remoção dos organismos do sedimento (KIKUCHI \& UIEDA, 1998), tornando-os mais disponíveis na coluna d'água e favorecendo sua utilização como alimento pela espécie. A predominância de insetos aquáticos na dieta de L. reinhardtii no período chuvoso também foi registrada por Pompeu \& Godinho (2003), em lagoas marginais no médio Rio São Francisco.

Espécies generalistas não possuem preferência acentuada por uma fonte alimentar, utilizando um amplo espectro de alimentos (AвelHa et al., 2001). Segundo os 
resultados obtidos, $L$. reinhardtii pode ser considerada como especialista no período da seca, de acordo com AMUNDSEN et al. (1996), na medida em que a abundância de presa específica foi superior a $80 \%$ em todos os trechos amostrados. Já no período chuvoso, a espécie apresenta uma tendência generalista, pois os valores de abundância relativa não se encontram equidistantes um do outro e além disto, os itens alimentares se apresentam mais diversificados que no período de seca.

Goulding (1980) afirma que peixes da família Anostomidae possuem hábitos alimentares generalistas e o tipo de alimento ingerido depende de sua disponibilidade no habitat. No entanto, a utilização dos recursos alimentares por estas espécies é limitada por adaptações morfofisiológicas de cada grupo (Melo \& RopKe, 2004).

A ocorrência de especialistas ou generalistas em determinado habitat é influenciada pela composição, disponibilidade e variabilidade espaço-temporal dos recursos alimentares (AвеLHa et al., 2001). Espécies especialistas são melhor sucedidas que generalistas quando há amplo suprimento de recursos e estes são renováveis (ODum, 1988). Tal fato pode ser evidenciado no reservatório de Sobradinho, onde existe uma grande disponibilidade de recursos renovados sazonalmente em função do pulso de inundação ao qual o reservatório é submetido. Portanto, considerando a teoria do forrageamento ótimo (BEGON et al., 2007), a espécie estudada se comporta como uma maximizadora de número, como a maioria dos peixes, consumindo as presas mais abundantes.

Em relação à amplitude de nicho, não existiu contribuição fenotípica em nenhum dos trechos, para ambos períodos, nem alto BPC (entre os componentes fenotípicos) e nem alto WPC (dentro do componente fenotípico) (AMUNDSEN et al., 1996). Em uma população com alta contribuição entre os componentes fenotípicos, diferentes indivíduos se especializam em diferentes tipos de recursos, enquanto numa população com alta contribuição dentro do componente fenotípico, a maioria dos indivíduos utiliza muitos tipos de recursos simultaneamente (AMUNDSEN et al., 1996). Isto não ocorreu no presente estudo, no qual a amplitude de nicho verificada para todos os ambientes foi restrita, sendo a maior registrada para o período de cheia, devido uma maior diversidade de itens encontrados.

Este comportamento especialista constatado para $L$. reinhardtii reflete a restrita amplitude de seu nicho trófico, principalmente no período de seca. Uma alta disponibilidade de recursos pode determinar uma diminuição na amplitude de nicho, uma vez que predadores tendem a se alimentar de presas mais abundantes no ambiente, visando maximizar o ganho energético (BEGON et al., 2007).

Dessa forma, conclui-se que L reinhardtii possui hábito onívoro no reservatório de Sobradinho, cujas categorias alimentares predominantes na dieta dependem do regime hidrológico do reservatório, a qual reflete em diferentes estratégias alimentares, como especialista no período de seca e generalista no período chuvoso, além de uma amplitude restrita de nicho trófico para a espécie.
Agradecimentos. Os autores agradecem à Companhia Hidro Elétrica do São Francisco (CHESF) e à Fundação Apolônio Salles de Desenvolvimento Educacional (FADURPE), pelo apoio logístico e financeiro. TNM, AAFR e NCLS agradecem à Coordenação de Aperfeiçoamento de Pessoal de Nível Superior (CAPES) e ao Conselho Nacional de Desenvolvimento Científico e Tecnológico (CNPq) pelas bolsas de doutorado. À Viviane Melo, pelo inestimável auxílio na identificação dos Chironomidae.

\section{REFERÊNCIAS BIBLIOGRÁFICAS}

Abelha, M. C. F.; Agostinho, A. A. \& Goulart, E. 2001. Plasticidade trófica em peixes de água doce. Acta Scientiarum, Biological Sciences 23(2):425-434.

Agostinho, A. A.; Gomes, L. C. \& Pelicice, F. M. 2007. Ecologia e manejo de recursos pesqueiros em reservatórios do Brasil. Maringá, Eduem. 501p.

Albrecht, M. P. \& Pellegrini-Caramaschi, E. 2003. Feeding ecology of Leporinus taeniofasciatus (Characiformes: Anostomidae) before and after installation of a hydroeletric plant in the upper rio Tocantins, Brazil. Neotropical Ichthyology 1(1):53-60.

Alvim, M. C. C. \& Peret, A. C. 2004. Food resource sustaining the fish fauna in a section of the Upper São Francisco River in Três Marias, MG, Brazil. Brazilian Journal of Biology 64(2):195-202.

Amundsen, P. A.; Gabler, H. M. \& Staldvik, F. J. 1996. A new approach to graphical analysis of feeding strategy from stomach contents data - mofification of the Costello (1990) method. Journal of Fish Biology 48:607-614.

Balassa, G. C.; Fugi, R.; Hahn, N. S. \& Galina, A. B. 2004. Dieta de espécies de Anostomidae (Teleostei, Characiformes) na área de influência do reservatório de Manso. Mato Grosso, Brasil. Iheringia, Série Zoologia 94(1):77-82.

Begon, M.; Townsend, C. R. \& Harper, J. L. 2007. Ecologia: de indivíduos a ecossistemas. 4ed. Porto Alegre, Artmed. 740p.

Bennemann, S. T.; Shibatta, O. A. \& Garavello, J. C. 2000. O alimento utilizado pelos peixes e sua disponibilidade no ambiente. In: Bennemann, S. T.; Shibatta, O. A. \& Garavello, J. C. eds. Peixes do rio Tibagi, uma abordagem ecológica. Londrina, Ed. UEL. p. 25-35.

Borges, H. L. F.; Melo, V. F.; Lima, E. L. A.; Queiroz, A. C. S. \& Severi, W. 2010. Caracterização da comunidade de macroinvertebrados bentônicos no reservatório de Sobradinho e submédio rio São Francisco. In: Moura, A. N.; Araújo, E. L.; Bittencourt-Oliveira, M. C.; Pimentel, R. M. M. \& Albuquerque, U. P. eds. Reservatórios do nordeste do Brasil: biodiversidade, ecologia e manejo. Bauru, Canal6. p.479-502.

Britski, H. A.; Birindelli, J. L. O. \& Garavello, J. C. 2012. A new species of Leporinus Agassiz, 1829 from the Upper Rio Paraná basin (Characiformes, Anostomidae) with redescription of L. elongatus Valenciennes, 1850 and L. obtusidens (Valenciennes, 1837). Papéis Avulsos de Zoologia 52(37):441-475.

Britski, H. A.; SAto, Y. \& RosA, A. B. S. 1984. Manual de identificação de peixes da região de Três Marias: com chaves de identificação para os peixes da bacia do São Francisco. 3ed. Brasília, Câmara dos Deputados/CODEVASF. 143p.

Buckup, P. A.; Menezes, N. A. \& Ghazzi, M. S. 2007. Catálogo das espécies de peixes de água doce do Brasil. Rio de Janeiro, Museu Nacional. 195p.

Clarke, K. R. \& Gorley, R. N. 2006. Primer v6: user manual/tutorial. Plymouth, Primer-E. 190p.

Costello, M. J. 1990. Predator feeding strategy and prey importance: a new graphical analysis. Journal of Fisf Biology 36:261-263.

Durães, R.; Pompeu, P. S. \& Godinho, A. L. 2001. Alimentação de quatro espécies de Leporinus (Characiformes, Anostomidae) durante a formação de um reservatório no sudeste do Brasil. Iheringia, Série Zoologia 90:183-191.

Ferreira, E. J. G. 1984. A ictiofauna da represa hidrelétrica de CuruáUna, Santarém, Pará II - Alimentação e hábitos alimentares das principais espécies. Amazoniana 9(1):1-16.

Garavello, J. C. \& Britski, H. A. 2003. Family Anostomidae. In: Reis, R. E.; Kullander, S. O. \& Ferraris, C. J. eds. Checklist of the freshwater fishes of south and central America. Porto Alegre, Edpucrs. p.71-84. 
Gerking, S. D. 1994. Feeding ecology of fish. California, Academic Press. 416p.

Gomes, J. H. C. \& Verani, J. R. 2003. Alimentação de peixes do reservatório de Três Marias. In: H. P. GodinHo. ed. Águas, peixes e pescadores do São Francisco das Minas Gerais. Belo Horizonte, PUC Minas. p. 195-227.

Goulding, M. 1980. The fishes and the forest: explorations in Amazonian natural history. Berkeley, University of California Press. 280p.

Hahn, N. S. \& Fugi, R. 2007. Alimentação de peixes em reservatórios brasileiros: alterações e conseqüências nos estágios iniciais do represamento. Oecologia Brasiliensis 11(4):469-480.

HurLbERT, S. H. 1978. The measurement of niche overlap and some relatives. Ecology 59(1):67-77.

HYsLOP, E. J. 1980. Stomach content analysis - a review of methods and their application. Journal of Fish Biology 17:411-429.

JunK, W. J.; Bayley, P. B. \& Sparks, R. E. 1989. The flood pulse concept in river - floodplain systems. In: Dodge, D. P. ed. Proceedings of the International Large River Symposium. Canadá, Canadian Fisheries - Annual Statistical Review. p.110-127.

Kikuchi, R. M. \& Uieda, V.S. 1998. Composição da comunidade de invertebrados de um ambiente lótico tropical e sua variação espacial e temporal. Oecologia Brasiliensis 1:157-173.

KREBS, C. J. 1999. Ecological Methodology. 2ed. Menlo Park, Benjamim/ Cummings. 634p.

Lowe-Mcconnell, R. 1999. Estudos ecológicos de comunidades de peixes tropicais. São Paulo, Edusp. 534p.

Melo, C. E. \& Ropke, C. P. 2004. Alimentação e distribuição de piaus (Pisces, Anostomidae) na Planície do Bananal, Mato Grosso, Brasil. Revista Brasileira de Zoologia 21(1):51-56.

Merritt, R. W. \& Cummins, K. W. 1996. An introduction to the aquatic insects of North America. Dubuque, Kendall/Hunt Publishing. 548p.

Nelson, J. S. 2006. Fishes of the world. 4ed. New York, John Wiley \& Sons. 601p.

Odum, E. P. 1988. Ecologia. Rio de Janeiro, Guanabara. 434p.

Oliveira, E. F. \& Goulart, E. 2000. Distribuição espacial de peixes em ambientes lênticos: interação de fatores. Acta Scientiarum, Biological Sciences 22(2):445-453.
Petry, A. C.; Thomaz, S. M. \& Esteves, F. A. 2011. Comunidade de peixes. In: Esteves, F. A. ed. Fundamentos de limnologia. 3ed. Rio de Janeiro, Interciência. p. 609-624.

Pinto, G. A.; Rocha, A. A. F.; Santos, N. C. L.; Medeiros, T. N. \& Severi, W. 2011. Variação sazonal na dieta de Triportheus guentheri (Garman, 1890) (Actinopterygii: Characidae), no reservatório de Sobradinho, rio São Francisco, BA. Boletim do Instituto de Pesca 37(3):295-306.

Pompeu, P. S. \& GodinHo, H. P. 2003. Ictiofauna de três lagoas marginais do médio São Francisco. In: Godinho, H. P. \& Godinho, A. L. eds. Águas, peixes e pescadores do São Francisco das Minas Gerais. Belo Horizonte, PUC Minas. p. 167-181.

Rocha, A. A. F.; Santos, N. C. L.; Medeiros, T. N.; Pinto, G. A. \& Severi, W. 2011. Diet composition and food overlap of Acestrorhynchus britskii and A. lacustris (Characiformes: Acestrorhynchidae) from Sobradinho reservoir, São Francisco river, Bahia State. Acta Scientiarum, Biological Sciences 33(4):407-415.

SidLAuSKAS, B. L. \& VARI, R. P. 2008. Phylogenetic relationships within South American fish family Anostomidae (Teleostei, Ostariophysi, Characiformes). Zoological Journal of the Linnean Society 154:70210.

Simone, L. R. L. 2006. Land and freshwater molluses of Brazil. São Paulo, EGB/FAPESP. 390p.

Sмiтн, D. G. 2001. Pennak's freshwater invertebrates of the United States: Porífera to Crustacea. New York, John Wiley. 638p.

Takeda, A. M.; Souza-Franco, G. J.; Melo, S. M. \& Monkolski, A. 2003. Invertebrados associados as macrófitas aquáticas da planície de inundação do alto rio Paraná, Brasil. In: Thomaz, S. M. \& BinI, L. M. eds. Ecologia e manejo de macrófitas aquáticas. Maringá, EDUEM. p. 243-260.

THORnTON, K. W. 1990. Perspectives on reservoir limnology. In: THORNTON, K. W.; Kimmel, B. L. \& Payne, F. E. eds. Reservoir limnology: ecological perspectives. New York, Wiley-Interscience. p. 1-13.

ZAVALA-CAMIN, L. A. 1996. Introdução aos estudos sobre alimentação natural em peixes. Maringá, EDUEM. 129p.

Recebido em 31 de outubro de 2013. Aceito em 28 de setembro de 2014. ISSN 0073-4721

Artigo disponível em: www.scielo.br/isz 